\title{
Discrete Painlevé Equations for Recurrence Coefficients of Laguerre-Hahn Orthogonal Polynomials of Class One
}

\author{
G. Filipuk ${ }^{a}$ and M.N. Rebocho ${ }^{b *}$ \\ ${ }^{a}$ Faculty of Mathematics, Informatics and Mechanics, University of Warsaw, Banacha 2, \\ Warsaw, 02-097, Poland; Institute of Mathematics, Polish Academy of Sciences, Sniadeckich 8, \\ Warsaw, 00-656, Poland. \\ ${ }^{b}$ Departmento de Matemática, Universidade da Beira Interior, 6201-001 Covilhã, Portugal; \\ CMUC, Apartado 3008, EC Santa Cruz, 3001-501 Coimbra, Portugal \\ (Received 00 Month 201X; final version received 0o Month 201X)
}

\begin{abstract}
In this paper we study recurrences for Laguerre-Hahn orthogonal polynomials of class one. It is shown for some families of such Laguerre-Hahn polynomials that the coefficients of the three term recurrence relation satisfy some forms of discrete Painlevé equations, namely, $d P_{I}$ and $d P_{I V}$.
\end{abstract}

Keywords: Orthogonal polynomials; Stieltjes functions; Riccati differential equations; difference equations; discrete Painlevé equations.

AMS Subject Classification: 33C45, 33E17, $42 \mathrm{C} 05$.

\section{Motivation}

Laguerre-Hahn orthogonal polynomials are related to Stieltjes functions, $S$, that satisfy a Riccati differential equation with polynomial coefficients [1-3]

$$
A S^{\prime}=B S^{2}+C S+D \text {. }
$$

The study of recurrences for orthogonal polynomials related to the differential equations of general type (1) is connected to a wide range of subjects in mathematics, such as probability theory [4], differential equations [5, 6], constructive approximation [7], etc. In particular, the semiclassical case - $B \equiv 0$ in (1) - has been much studied in the literature due to the well-known connections with integrable systems and Painlevé equations (see, amongst many others, $[8,9])$.

In the literature of orthogonal polynomials, the study of Laguerre-Hahn orthogonal polynomials proceeds in two directions. One of them is the study within the framework of modifications of measures and the analysis of the corresponding perturbations on the sequences of orthogonal polynomials. Indeed, Laguerre-Hahn families of orthogonal polynomials may appear as a result of modifications of semi-classical orthogonal polynomials, for example, through some perturbations of the coefficients of the three

\footnotetext{
* Corresponding author. Email: mneves@ubi.pt
} 
term recurrence relation [10-13], or through spectral rational modifications of the Stieltjes functions [14-16]. Another direction of study concerns the problem of classification of families of orthogonal polynomials in terms of classes of differential equations (1), which amounts to the classification in terms of classes of distributional equations for the corresponding linear functionals. In more specific terms, given information on the polynomial coefficients of (1), the goal is to describe the systems of equations for the recurrence relation coefficients of the corresponding sequence of orthogonal polynomials, the so-called Laguerre-Freud equations [17-21]. Generally, such equations are given in terms of non-linear systems, whose complexity increases with the degrees of the polynomials in (1). In this topic one should emphasize the vast literature on the semi-classical case, showing that recurrence coefficients of semi-classical orthogonal polynomials can often be expressed in terms of solutions of Painlevé equations (see, amongst many others, $[8,9,22,23])$.

In the present work we focus on the difference equations for the recurrence relation coefficients of Laguerre-Hahn orthogonal polynomials in class one (cf. Section 2), $\max \{\operatorname{deg}(A), \operatorname{deg}(B)\} \leq 3, \operatorname{deg}(C)=2$ or $\max \{\operatorname{deg}(A), \operatorname{deg}(B)\}=3, \operatorname{deg}(C) \leq 2$ in (1). The symmetric case has been analysed in [24], but the problem of a classification of such a class of orthogonal polynomials remains open. Our main results are contained in Section 3, in Theorems 3.3, 3.6, and 3.8. We focus on $\operatorname{deg}(A) \leq 1$ in $(1)$, and show that the recurrence relation coefficients of orthogonal polynomials belonging to Laguerre-Hahn class one in the non-symmetric case related to $\operatorname{deg}(A) \leq 1$ in (1) are governed by difference equations of the Painlevé type, namely, $d P_{I V}$ and $d P_{I}$. The expressions of discrete Painlevé equations in terms of the coefficients of the Riccati equation for the Stieltjes function are deduced. The main tool is the so-called structure relations for LaguerreHahn polynomials [1, Eqs. (3.9), (3.10)] which have been recently re-interpreted and studied within the theory of matrix Sylvester equations in [14, Theorem 1]. Comments on the analysis of the cases $\operatorname{deg}(A) \geq 2$ are given in Remark 3.9.

The rest of the paper is organized as follows. In Section 2 we give preliminary results on Laguerre-Hahn orthogonal polynomials. Section 3 is devoted to the derivation of recurrences for Laguerre-Hahn orthogonal polynomials and to show the connection to the discrete Painleve equation $d P_{I V}$ and $d P_{I}$. In Section 4 we illustrate our main results with examples of modified Laguerre and associated Laguerre polynomials.

\section{Preliminary results}

Let $\mathbb{P}=\operatorname{span}\left\{x^{k}: k \in \mathbb{N}_{0}\right\}$ be a linear space of polynomials with complex coefficients and let $\mathbb{P}^{\prime}$ be its algebraic dual space. We will denote the action of $u \in \mathbb{P}^{\prime}$ on $f \in \mathbb{P}$ by $\langle u, f\rangle$.

Given the moments of $u, u_{n}=\left\langle u, x^{n}\right\rangle, n \geq 0$, where we take $u_{0}=1$, the principal leading minors of the corresponding Hankel matrix are defined by $H_{n}=\operatorname{det}\left(u_{i+j}\right)_{i, j=0}^{n}, n \geq$ 0 , where, by a convention, $H_{-1}=1$. The functional $u$ is said to be quasi-definite (respectively, positive-definite) if $H_{n} \neq 0$ (respectively, $H_{n}>0$ ), for all $n \geq 0$.

Definition 2.1. (see [25]) Let $u \in \mathbb{P}^{\prime}$ and let $\left\{P_{n}\right\}_{n \geq 0}$ be a sequence of polynomials such that $\operatorname{deg}\left(P_{n}\right)=n, n \geq 0 .\left\{P_{n}\right\}_{n \geq 0}$ is said to be a sequence of orthogonal polynomials with respect to $u$ if

$$
\left\langle u, P_{n} P_{m}\right\rangle=h_{n} \delta_{n, m}, h_{n} \neq 0, \quad n, m \geq 0
$$

Throughout the paper we shall take each $P_{n}$ monic, that is, $P_{n}(x)=x^{n}+$ lower degree terms, and we will abbreviate a sequence of monic orthogonal polynomials $\left\{P_{n}\right\}_{n \geq 0}$ by 


\section{SMOP.}

The equivalence between the quasi-definiteness of $u \in \mathbb{P}^{\prime}$ and the existence of a SMOP with respect to $u$ is well known in the literature on orthogonal polynomials (see [25, 26]). Furthermore, if $u$ is positive-definite, then it has an integral representation in terms of a positive Borel measure $\mu$ supported on an infinite point set $I$ of the real line such that

$$
\left\langle u, x^{n}\right\rangle=\int_{I} x^{n} d \mu(x), n \geq 0,
$$

and the orthogonality condition (2) becomes

$$
\int_{I} P_{n}(x) P_{m}(x) d \mu(x)=h_{n} \delta_{n, m}, h_{n}>0, \quad n, m \geq 0 .
$$

If $\mu$ is an absolutely continuous measure supported on $I$, and $w$ denotes its RadonNikodym derivative with respect to the Lebesgue measure, i.e. $d \mu(x)=w(x) d x$, then we will also say that $\left\{P_{n}\right\}_{n \geq 0}$ is orthogonal with respect to $w$.

Monic orthogonal polynomials satisfy a three term recurrence relation [25]

$$
P_{n+1}(x)=\left(x-\beta_{n}\right) P_{n}(x)-\gamma_{n} P_{n-1}(x), \quad n=1,2, \ldots
$$

with $P_{0}(x)=1, P_{1}(x)=x-\beta_{0}$ and $\gamma_{n} \neq 0, n \geq 1, \gamma_{0}=1$.

Definition 2.2. (see [26]) Let $\left\{P_{n}\right\}_{n \geq 0}$ be a $S M O P$ with respect to $u \in \mathbb{P}^{\prime}$. The sequence of monic associated polynomials of the first kind is defined by

$$
P_{n}^{(1)}(x)=\left\langle u_{t}, \frac{P_{n+1}(x)-P_{n+1}(t)}{x-t}\right\rangle, \quad n \geq 0,
$$

where $u_{t}$ denotes the action of $u$ on the variable $t$.

The sequence $\left\{P_{n}^{(1)}\right\}_{n \geq 0}$ also satisfies a three term recurrence relation

$$
P_{n}^{(1)}(x)=\left(x-\beta_{n}\right) P_{n-1}^{(1)}(x)-\gamma_{n} P_{n-2}^{(1)}(x), \quad n=1,2, \ldots
$$

with $P_{-1}^{(1)}(x)=0, P_{0}^{(1)}(x)=1$.

Definition 2.3. The Stieltjes function of $u \in \mathbb{P}^{\prime}$ is defined by $S(x)=\sum_{n=0}^{+\infty} \frac{u_{n}}{x^{n+1}}$, where $u_{n}$ are the moments of $u$.

The function $S$ has a continued fraction expansion given by

$$
S(x)=\frac{1}{x-\beta_{0}-\frac{\gamma_{1}}{x-\beta_{1}-\frac{\gamma_{2}}{\ddots}}}
$$

where the $\gamma$ 's and the $\beta$ 's are the coefficients in the three term recurrence relation for the corresponding SMOP. Note that if $u$ is positive-definite, defined by (3), then $S$ is the so-called Borel or Stieltjes transform of the measure,

$$
S(x)=\int_{I} \frac{d \mu(t)}{x-t}, \quad x \in \mathbb{C} \backslash I .
$$


In account of (6), the Stieltjes functions corresponding to $\left\{P_{n}\right\}_{n \geq 0}$ and $\left\{P_{n}^{(1)}\right\}_{n \geq 0}$, denoted by $S$ and $S^{(1)}$, respectively, are related by [16]

$$
\gamma_{1} S^{(1)}(x)=-\frac{1}{S(x)}+\left(x-\beta_{0}\right)
$$

Definition 2.4. (see [3]) A Stieltjes function $S$ and the corresponding linear functional $u$, are said to be Laguerre-Hahn if there exist polynomials $A, B, C, D$, such that $S$ satisfies a Riccati differential equation

$$
A S^{\prime}=B S^{2}+C S+D, \quad A \neq 0
$$

A sequence of orthogonal polynomials related to $S$ (or $u$ ) is then called Laguerre-Hahn. If $B=0$, then $S$ (or u) is said to be semi-classical.

Eq. (8) is equivalent to the distributional equation [1, Theorem 3.1]

$$
\mathcal{D}(A u)=\psi u+B\left(x^{-1} u^{2}\right), \quad \psi=A^{\prime}+C .
$$

The polynomial $D$ can be written in terms of $A, B, C$ as

$$
D(x)=-\left(u \theta_{0} A\right)^{\prime}(x)+\left(u \theta_{0}\left(A^{\prime}+C\right)\right)(x)+\left(u^{2} \theta_{0}^{2} B\right)(x) .
$$

The definition of $\theta_{0} p, p \in \mathbb{P}$, as well as the right product of a linear functional by a polynomial, and the product of two linear functionals, can by found in $[1,3]$.

Remark 2.5. Let $B \equiv 0$. If $\operatorname{deg}(\psi)=1, \operatorname{deg}(A) \leq 2$, then $u$ is called a classical functional, and the corresponding orthogonal polynomials are the so-called classical orthogonal polynomials, that is, Hermite, Laguerre, Jacobi and Bessel polynomials. $\operatorname{deg}(\psi) \geq 1$ corresponds to the semi-classical case.

If $u$ is positive-definite, defined in terms of a weight $w$, then the semi-classical character of $u$, that is, $\mathcal{D}(A u)=\psi u$ or $A S^{\prime}=C S+D$, is equivalent to the Pearson equation $A w^{\prime}=C w$, with $w$ satisfying the boundary conditions [3]

$$
\left.x^{n} A(x) w(x)\right|_{a, b}=0, \quad n \geq 0,
$$

where $a, b$ (eventually $a$ or $b$ infinite) are linked with the roots of $A$. In such a case, $w$ is the weight function on the support $I=[a, b]$.

In the semi-classical case, integral representations for $u$ are known $[27,28]$. Let us emphasize that the problem of the integral representation of Laguerre-Hahn linear functionals remains an open problem.

Note that the triple $(A, \psi, B)$ satisfying $(9)$ is not unique, indeed, many triples of polynomials can be associated with such a distributional equation, but only one canonical set of minimal degree exists. To a Laguerre-Hahn linear functional one associates the class, a non-negative integer, defined as follows.

Definition 2.6. [3] The class of the linear functional u satisfying (9) is the minimum value of $\max \{\operatorname{deg}(\psi)-1, d-2\}, d=\max \{\operatorname{deg}(A), \operatorname{deg}(B)\}$, for all triples of polynomials $(A, \psi, B)$ satisfying $(9)$.

The functional $u$ related to the Riccati equation $A S^{\prime}=B S^{2}+C S+D$ is of class $s=\max \{\operatorname{deg}(C)-1, d-2\}$ if, and only if, the polynomials $A, B, C$ and $D$ have no common zeroes [24, Prop. 2.5]. 
The recurrence relations (4) and (5) can be written in the matrix form

$$
Y_{n}=\mathcal{A}_{n} Y_{n-1}, \quad Y_{n}=\left[\begin{array}{cc}
P_{n+1} & P_{n}^{(1)} \\
P_{n} & P_{n-1}^{(1)}
\end{array}\right], \quad \mathcal{A}_{n}=\left[\begin{array}{cc}
x-\beta_{n}-\gamma_{n} \\
1 & 0
\end{array}\right], \quad n \geq 1
$$

with initial conditions $Y_{0}=\left[\begin{array}{cr}x-\beta_{0} & 1 \\ 1 & 0\end{array}\right]$. The matrix $\mathcal{A}_{n}$ is known as the transfer matrix.

Theorem 2.7. (see [14]) Let $S$ be a Stieltjes function, let $\left\{Y_{n}\right\}_{n \geq 0}$ be the corresponding sequence defined in (10), and let $\beta_{n}, \gamma_{n}$ be the coefficients in the recurrence relation. The following statements are equivalent:

(a) S satisfies (8),

$$
A S^{\prime}=B S^{2}+C S+D, A, B, C, D \in \mathbb{P}
$$

(b) $Y_{n}$ satisfies the matrix Sylvester equation

$$
A Y_{n}^{\prime}=\mathcal{B}_{n} Y_{n}-Y_{n} \mathcal{C}, \quad n \geq 0,
$$

where

$$
\mathcal{B}_{n}=\left[\begin{array}{cc}
l_{n} & \Theta_{n} \\
-\Theta_{n-1} / \gamma_{n} & l_{n-1}+\left(x-\beta_{n}\right) \Theta_{n-1} / \gamma_{n}
\end{array}\right], \mathcal{C}=\left[\begin{array}{cc}
C / 2 & -D \\
B & -C / 2
\end{array}\right]
$$

with $l_{n}, \Theta_{n}$ polynomials of uniformly bounded degrees, satisfying the initial conditions

$$
\Theta_{-1}=D, \Theta_{0}=A+\left(x-\beta_{0}\right)\left(C / 2-l_{0}\right)+B, l_{-1}=C / 2, l_{0}=-C / 2-\left(x-\beta_{0}\right) D
$$

(c) the transfer matrix $\mathcal{A}_{n}=\left[\begin{array}{cc}x-\beta_{n} & -\gamma_{n} \\ 1 & 0\end{array}\right]$ satisfies the matrix Sylvester equation

$$
A \mathcal{A}_{n}^{\prime}=\mathcal{B}_{n} \mathcal{A}_{n}-\mathcal{A}_{n} \mathcal{B}_{n-1}, \quad n \geq 1
$$

Remark 2.8. If the class of the linear functional related to (8) is $s$, the polynomials $l_{n}, \Theta_{n}$ in $\mathcal{B}_{n}$ satisfy $\operatorname{deg}\left(l_{n}\right)=s+1, \operatorname{deg}\left(\Theta_{n}\right)=s[1]$.

Let us emphasize that Eq. (13) is obtained from the compatibility for the Lax pair

$$
\left\{\begin{array}{l}
Y_{n}=\mathcal{A}_{n} Y_{n-1} \\
A Y_{n}^{\prime}=\mathcal{B}_{n} Y_{n}-Y_{n} \mathcal{C}, \quad n \geq 1 .
\end{array}\right.
$$

Furthermore, we have [14, Corollary 1]

$$
\begin{gathered}
\operatorname{tr} \mathcal{B}_{n}=0, \quad n \geq 0, \\
\operatorname{det} \mathcal{B}_{n}=\operatorname{det} \mathcal{B}_{0}+A \sum_{k=1}^{n} \frac{\Theta_{k-1}}{\gamma_{k}}, \quad n \geq 1,
\end{gathered}
$$

with $\operatorname{det} \mathcal{B}_{0}=D(A+B)-(C / 2)^{2}$. 
Eq. (13) yields two non-trivial equations, respectively, from positions $(1,1)$ and $(1,2)$ :

$$
\begin{aligned}
& A=\left(x-\beta_{n}\right)\left(l_{n}-l_{n-1}\right)+\Theta_{n}-\gamma_{n} \frac{\Theta_{n-2}}{\gamma_{n-1}}, \\
& l_{n}+\left(x-\beta_{n}\right) \frac{\Theta_{n-1}}{\gamma_{n}}=l_{n-2}+\left(x-\beta_{n-1}\right) \frac{\Theta_{n-2}}{\gamma_{n-1}} .
\end{aligned}
$$

Note that, after some basic computations, (17) implies (16) and (18) implies (15).

\section{Recurrences for Laguerre-Hahn orthogonal polynomials of class $s=1$}

The Stieltjes functions $S$ associated with the Laguerre-Hahn orthogonal polynomials of class $s=1$ satisfy $A S^{\prime}=B S^{2}+C S+D$ with $\operatorname{deg}(D)=1$ and

$$
\max \{\operatorname{deg}(A), \operatorname{deg}(B)\} \leq 3, \operatorname{deg}(C)=2,
$$

or

$$
\max \{\operatorname{deg}(A), \operatorname{deg}(B)\}=3, \operatorname{deg}(C)<2 .
$$

One of the key ingredients in the sequel is the differential system (11). This is the matrix form of the so-called structure relations

$$
\begin{gathered}
A\left(P_{n}^{(1)}\right)^{\prime}=D P_{n+1}+\left(l_{n}+C / 2\right) P_{n}^{(1)}+\Theta_{n} P_{n-1}^{(1)}, \quad n \geq 0, \\
A P_{n+1}^{\prime}=\left(l_{n}-C / 2\right) P_{n+1}-B P_{n}^{(1)}+\Theta_{n} P_{n}, \quad n \geq 0,
\end{gathered}
$$

(see $[1$, Eqs. $(3.9),(3.10)]$ ) with the polynomials $l_{n}, \Theta_{n}$ satisfying $\operatorname{deg}\left(l_{n}\right)=2, \operatorname{deg}\left(\Theta_{n}\right)=$ 1 .

Remark 3.1. In the semi-classical case, that is, $B \equiv 0$, Eq. (20) becomes $A P_{n+1}^{\prime}=$ $\left(l_{n}-C / 2\right) P_{n+1}+\Theta_{n} P_{n}$, which can be comparable to [29, Eq. (1.5)].

Lemma 3.2. Let $S$ be a Stieltjes function satisfying $A S^{\prime}=B S^{2}+C S+D$. Let $\left\{P_{n}\right\}_{n \geq 0}$ be the $S M O P$ associated with $S$, satisfying the recurrence relation (4),

$$
P_{n+1}(x)=\left(x-\beta_{n}\right) P_{n}(x)-\gamma_{n} P_{n-1}(x), \quad n=0,1,2, \ldots
$$

Set

$$
\begin{gathered}
A(x)=a_{3} x^{3}+a_{2} x^{2}+a_{1} x+a_{0}, \quad B(x)=b_{3} x^{3}+b_{2} x^{2}+b_{1} x+b_{0}, \\
C(x)=c_{2} x^{2}+c_{1} x+c_{0}, \quad D(x)=d_{1} x+d_{0}, \\
l_{n}(x)=\ell_{n, 2} x^{2}+\ell_{n, 1} x+\ell_{n, 0}, \quad \Theta_{n}(x)=\theta_{n, 1} x+\theta_{n, 0} .
\end{gathered}
$$

With the functions defined above, we have

$$
\begin{aligned}
& d_{1}=-a_{3}-b_{3}-c_{2}, \\
& d_{0}=-\left(2 a_{3}+2 b_{3}+c_{2}\right) \beta_{0}-a_{2}-b_{2}-c_{1}, \\
& \ell_{n, 2}=(n+1) a_{3}+b_{3}+c_{2} / 2, \\
& \ell_{n, 1}=a_{3}\left(\eta_{n}+\beta_{0}\right)+(n+1) a_{2}+b_{3} \beta_{0}+b_{2}+c_{1} / 2,
\end{aligned}
$$


where

$$
\begin{aligned}
\ell_{n, 0}= & \lambda_{n, 0}-\theta_{n, 1} \\
\lambda_{n, 0}= & -2 a_{3}\left(\nu_{n}+\beta_{0} \eta_{n}-\gamma_{1}\right)+(n+1) a_{1}+b_{3}\left(\gamma_{1}+\beta_{0}^{2}\right)+b_{2} \beta_{0}+b_{1}+c_{0} / 2 \\
& +\left(\eta_{n}+\beta_{0}\right)\left(a_{3}\left(\eta_{n}+\beta_{0}\right)+a_{2}\right) \\
\theta_{n, 1}= & -\gamma_{n+1}\left((2 n+3) a_{3}+2 b_{3}+c_{2}\right) \\
\theta_{n, 0}= & -\gamma_{n+1}\left\{2 a_{3}\left(\eta_{n}+(n+2) \beta_{n+1}+\beta_{0}\right)+a_{2}(2 n+3)+2 b_{3}\left(\beta_{0}+\beta_{n+1}\right)\right. \\
& \left.+2 b_{2}+c_{2} \beta_{n+1}+c_{1}\right\}
\end{aligned}
$$

with

$$
\eta_{n}=\sum_{k=1}^{n} \beta_{k}, \quad \nu_{n}=\sum_{1 \leq i<j \leq n}^{n} \beta_{i} \beta_{j}-\sum_{k=2}^{n} \gamma_{k}, \quad n \geq 1 .
$$

Also, we have

$$
a_{1}+b_{1}+c_{0}+\left(2 a_{2}+2 b_{2}+c_{1}\right) \beta_{0}+\left(3 a_{3}+3 b_{3}+c_{2}\right) \beta_{0}^{2}+\left(3 a_{3}+2 b_{3}+c_{2}\right) \gamma_{1}=0 .
$$

Proof. In account of (4) and (5),

$$
\begin{aligned}
P_{n}^{(1)}(x) & =x^{n}-\eta_{n} x^{n-1}+\nu_{n} x^{n-2}+\cdots \\
P_{n+1}(x) & =x^{n+1}-\left(\eta_{n}+\beta_{0}\right) x^{n}+\left(\nu_{n}+\beta_{0} \eta_{n}-\gamma_{1}\right) x^{n-1}+\cdots
\end{aligned}
$$

Eqs. (21) and (22) follow by equating the coefficients of $x^{n+2}$ and $x^{n+1}$, respectively, in (19). Eqs. (23) - (25) follow by equating the coefficients of $x^{n+3}, x^{n+2}$, and $x^{n+1}$, respectively, in (20). Eqs. (27) - (28) follow by equating the coefficients of $x^{2}$ and $x$, respectively, in (15), $l_{n}(x)+l_{n-1}(x)+\left(x-\beta_{n}\right) \frac{\Theta_{n-1}(x)}{\gamma_{n}}=0$. Eq. (30) follows by equating the coefficients of $x^{n}$ in (19).

\subsection{Discrete Painlevé equations for Laguerre-Hahn Stieltjes functions}

We will use two key ingredients to deduce recurrences for the Laguerre-Hahn polynomials: the formula for the trace and the determinant of the matrix $\mathcal{B}_{n}$, Eqs. (15) and (16), defining the Lax pair (14).

From (15), equivalently, $l_{n}(x)+l_{n-1}(x)+\left(x-\beta_{n}\right) \frac{\Theta_{n-1}(x)}{\gamma_{n}}=0, n \geq 0$, we get

$$
\begin{gathered}
l_{n}\left(\beta_{n}\right)+l_{n-1}\left(\beta_{n}\right)=0, \\
\frac{l_{n}(0)+l_{n-1}(0)}{\beta_{n}}=\frac{\Theta_{n-1}(0)}{\gamma_{n}}, \quad \beta_{n} \neq 0 .
\end{gathered}
$$

From (16) we get, for every $\alpha$ such that $A(\alpha)=0$,

$$
\operatorname{det} \mathcal{B}_{n-1}(\alpha)=\operatorname{det} \mathcal{B}_{0}(\alpha)
$$


hence, taking into account $\operatorname{det} \mathcal{B}_{n}=-l_{n}^{2}+\Theta_{n} \frac{\Theta_{n-1}}{\gamma_{n}}$, we have

$$
\Theta_{n-1}(\alpha) \frac{\Theta_{n-2}(\alpha)}{\gamma_{n-1}}=\operatorname{det} \mathcal{B}_{0}(\alpha)+l_{n-1}^{2}(\alpha)
$$

Therefore, for $\alpha$ such that $A(\alpha)=0$,

$$
\frac{\Theta_{n-1}(\alpha)}{\gamma_{n}} \frac{\Theta_{n-2}(\alpha)}{\gamma_{n-1}}=\frac{\operatorname{det}\left(\mathcal{B}_{0}\right)(\alpha)+\left(l_{n-1}(\alpha)\right)^{2}}{\gamma_{n}}
$$

Eqs. (31) - (33) will be used in the proof of the next Theorem.

Theorem 3.3. Let $S$ be a Stieltjes function satisfying $A S^{\prime}=B S^{2}+C S+D$ with

$$
\begin{gathered}
A(x)=a_{1} x, B(x)=b_{3} x^{3}+b_{2} x^{2}+b_{1} x+b_{0}, \\
C(x)=c_{2} x^{2}+c_{1} x+c_{0}, D(x)=d_{1} x+d_{0},
\end{gathered}
$$

where $d_{1}$ and $d_{0}$ are given by (21) and (22), respectively. Let $\left\{P_{n}\right\}_{n \geq 0}$ be the SMOP associated with $S$, satisfying the recurrence relation (4),

$$
P_{n+1}(x)=\left(x-\beta_{n}\right) P_{n}(x)-\gamma_{n} P_{n-1}(x), \quad n=0,1,2, \ldots,
$$

with $\beta_{n} \neq 0, n=0,1,2, \ldots$

Set $\eta=2 b_{3}+c_{2}, \mu=b_{3}\left(\gamma_{1}+\beta_{0}^{2}\right)+b_{2} \beta_{0}+b_{1}+c_{0} / 2, \lambda=2 b_{3} \beta_{0}+2 b_{2}+c_{1}, \xi_{0}=c_{0}^{2} / 4-d_{0} b_{0}$. The following statements hold:

(a) Let $\eta<0$. The sequences $\left\{x_{n}\right\}_{n=0}^{\infty}$ and $\left\{y_{n}\right\}_{n=0}^{\infty}$ expressed in terms of the coefficients in the three term recurrence relation by

$$
x_{n}=\frac{\sqrt{-\eta}}{\eta \beta_{n}+\lambda}, \quad y_{n}=-\left(\eta \gamma_{n}+n a_{1}+\mu\right)
$$

satisfy the following discrete system of Painlevé equations $d P_{I V}$ :

$$
x_{n-1} x_{n}=\frac{y_{n}+n a_{1}+\mu}{y_{n}^{2}-\xi_{0}}, \quad y_{n}+y_{n+1}=\frac{1}{x_{n}}\left(\frac{\lambda}{\sqrt{-\eta}}-\frac{1}{x_{n}}\right),
$$

with the initial conditions $x_{0}=\frac{\sqrt{-\eta}}{\eta \beta_{0}+\lambda}, y_{0}=-(\eta+\mu)$.

(b) Let $\eta>0$. The sequences $\left\{x_{n}\right\}_{n=0}^{\infty}$ and $\left\{y_{n}\right\}_{n=0}^{\infty}$ expressed in terms of the coefficients in the three term recurrence relation by

$$
x_{n}=\frac{\sqrt{\eta}}{\eta \beta_{n}+\lambda}, \quad y_{n}=\eta \gamma_{n}+n a_{1}+\mu
$$

satisfy the following discrete system of Painlevé equations $d P_{I V}$ :

$$
x_{n-1} x_{n}=\frac{y_{n}-n a_{1}-\mu}{y_{n}^{2}-\xi_{0}}, \quad y_{n}+y_{n+1}=\frac{1}{x_{n}}\left(\frac{\lambda}{\sqrt{\eta}}-\frac{1}{x_{n}}\right),
$$

with the initial conditions $x_{0}=\frac{\sqrt{\eta}}{\eta \beta_{0}+\lambda}, y_{0}=\eta+\mu$. 
Proof. Let us show how to deduce equations in case (a). Case (b) is similar.

Eq. (31) gives us, under the notation of Lemma 3.2,

$$
\left(\ell_{n, 2} \beta_{n}^{2}+\ell_{n, 1} \beta_{n}+\ell_{n, 0}\right)+\left(\ell_{n-1,2} \beta_{n}^{2}+\ell_{n-1,1} \beta_{n}+\ell_{n-1,0}\right)=0 .
$$

Taking into account the formulae for $\ell_{n, 2}, \ell_{n, 1}$ given in (23) and (24), the above equation yields

$$
\eta \beta_{n}^{2}+\lambda \beta_{n}=-\ell_{n-1,0}-\ell_{n, 0}, \quad \eta=2 b_{3}+c_{2}, \lambda=2 b_{3} \beta_{0}+2 b_{2}+c_{1},
$$

thus,

$$
\beta_{n}\left(\eta \beta_{n}+\lambda\right)=-l_{n-1}(0)-l_{n}(0)
$$

Therefore, we obtain, for $\beta_{n} \neq 0$,

$$
\left(\eta \beta_{n}+\lambda\right)\left(\eta \beta_{n-1}+\lambda\right)=\frac{\left(l_{n-1}(0)+l_{n}(0)\right)}{\beta_{n}} \frac{\left(l_{n-2}(0)+l_{n-1}(0)\right)}{\beta_{n-1}} .
$$

In account of (32), the equation above can be written as

$$
\left(\eta \beta_{n}+\lambda\right)\left(\eta \beta_{n-1}+\lambda\right)=\frac{\Theta_{n-1}(0)}{\gamma_{n}} \frac{\Theta_{n-2}(0)}{\gamma_{n-1}}
$$

In account of (33) with $\alpha=0\left(\right.$ as $\left.A(x)=a_{1} x\right)$, Eq. (40) can be written as

$$
\left(\eta \beta_{n}+\lambda\right)\left(\eta \beta_{n-1}+\lambda\right)=\frac{\left(l_{n-1}(0)\right)^{2}-\xi_{0}}{\gamma_{n}}
$$

with $\xi_{0}=-\operatorname{det} \mathcal{B}_{0}(0)$.

Note that, as $l_{n}(0)=\eta \gamma_{n+1}+(n+1) a_{1}+\mu, \quad \mu=b_{3}\left(\gamma_{1}+\beta_{0}^{2}\right)+b_{2} \beta_{0}+b_{1}+c_{0} / 2$, then (39) yields

$$
\beta_{n}\left(\eta \beta_{n}+\lambda\right)=-\left(\eta \gamma_{n}+n a_{1}+\mu\right)-\left(\eta \gamma_{n+1}+(n+1) a_{1}+\mu\right) .
$$

By defining $x_{n}, y_{n}$ as in (35),

$$
x_{n}=\frac{\sqrt{-\eta}}{\eta \beta_{n}+\lambda}, \quad y_{n}=-\left(\eta \gamma_{n}+n a_{1}+\mu\right)
$$

we obtain that Eqs. (41) and (42) are, respectively, the first and second equations given in (36). Indeed, let us write Eq. (41) in its equivalent form

$$
\frac{\sqrt{-\eta}}{\left(\eta \beta_{n}+\lambda\right)} \frac{\sqrt{-\eta}}{\left(\eta \beta_{n-1}+\lambda\right)}=\frac{-\eta \gamma_{n}}{\left(l_{n-1}(0)\right)^{2}-\xi_{0}}
$$

that is, we have $x_{n-1} x_{n}=\frac{y_{n}+n a_{1}+\mu}{y_{n}^{2}-\xi_{0}}$. Also, let us write the left-hand side of (42) as

$$
\frac{\eta \beta_{n}+\lambda}{\sqrt{-\eta}}\left(\frac{\lambda}{\sqrt{-\eta}}-\frac{\eta \beta_{n}+\lambda}{\sqrt{-\eta}}\right) .
$$


Then, (42) reads as $\frac{1}{x_{n}}\left(\frac{\lambda}{\sqrt{-\eta}}-\frac{1}{x_{n}}\right)=y_{n}+y_{n+1}$.

Remark 3.4. Recall that $B \equiv 0$ in Theorem 3.3 gives Painlevé equations for particular families of semi-classical orthogonal polynomials of class $s=1$.

The case $A(x) \equiv 0$, that is, $a_{1}=0$ in (34), concerns a second degree equation $B S^{2}+$ $C S+D=0$. In such a case, $S$ is related to the so-called family of second degree forms [30].

Remark 3.5. Equations (36) and (38) can be obtained as a limiting case of the asymmetric Painlevé equation $d P_{I V}[31$, Eq. (1.2)] given by

$$
u_{n} u_{n-1}=\frac{a\left(v_{n}+z_{n}-b\right)}{v_{n}^{2}-\gamma^{2}}, \quad v_{n}+v_{n+1}=\frac{c}{u_{n}}+\frac{z_{n+1 / 2}+d}{u_{n}-1}
$$

with $z_{n}=\alpha_{1} n+\beta_{1}$. Indeed, by taking $b=0, u_{n}=x_{n} / \varepsilon, v_{n}=\varepsilon y_{n}, z_{n}=\varepsilon\left(\mu+n a_{1}\right)$, $a=1 / \varepsilon, \gamma=\varepsilon / \sqrt{\xi_{0}}, c=1 / \varepsilon+\lambda / \sqrt{-\eta}, d=-1 / \varepsilon$ and letting $\varepsilon$ tend to zero we obtain (36). For (38) the calculations are similar.

3.1.1 The case $A(x)=a_{0} \neq 0$.

Let us emphasize that the technique used in Theorem 3.3 to deduce the difference equations for the coefficients $\beta_{n}, \gamma_{n}$ cannot be applied to the case $A(x)=a_{0} \neq 0$. Indeed, (31) yields (39), with $l_{n}(0)=\eta \gamma_{n+1}+\mu$, and such an equation implies (40). However, in the present case, (40) does not give new identities and (41) does not hold, as $A(0) \neq 0$.

The difference equations concerning the case $A(x)=a_{0} \neq 0$ are given in the theorem that follows.

Theorem 3.6. Let $S$ be a Stieltjes function satisfying $A S^{\prime}=B S^{2}+C S+D$ with

$$
\begin{gathered}
A(x)=a_{0} \neq 0, B(x)=b_{3} x^{3}+b_{2} x^{2}+b_{1} x+b_{0}, \\
C(x)=c_{2} x^{2}+c_{1} x+c_{0}, D(x)=d_{1} x+d_{0},
\end{gathered}
$$

where $d_{1}$ and $d_{0}$ are given by (21) and (22), respectively. Let $\left\{P_{n}\right\}_{n \geq 0}$ be the SMOP associated with $S$, satisfying the recurrence relation (4),

$$
P_{n+1}(x)=\left(x-\beta_{n}\right) P_{n}(x)-\gamma_{n} P_{n-1}(x), \quad n=0,1,2, \ldots,
$$

with $\beta_{n} \neq 0, n=0,1,2, \ldots$

Set $\eta=2 b_{3}+c_{2}, \mu=b_{3}\left(\gamma_{1}+\beta_{0}^{2}\right)+b_{2} \beta_{0}+b_{1}+c_{0} / 2, \lambda=2 b_{3} \beta_{0}+2 b_{2}+c_{1}$. The recurrence relation coefficients $\beta_{n}, \gamma_{n}$ are related through the following discrete system:

$$
\begin{aligned}
& \beta_{n}\left(\eta \beta_{n}+\lambda\right)=-\left(\eta \gamma_{n}+\mu\right)-\left(\eta \gamma_{n+1}+\mu\right), \quad n \geq 0, \\
& \eta\left(\beta_{n}+\beta_{n+1}\right)+\lambda=\frac{-n a_{0}+\gamma_{1}\left(\eta\left(\beta_{0}+\beta_{1}\right)+\lambda\right)}{\gamma_{n+1}}, \quad n \geq 1 .
\end{aligned}
$$

Moreover, the sequences

$$
x_{n}=\eta \beta_{n}+\lambda / 2, \quad z_{n}=\eta^{2}\left(-n a_{0}+\gamma_{1}\left(\eta\left(\beta_{0}+\beta_{1}\right)+\lambda\right)\right)
$$

satisfy the alternative discrete Painlevé equation $d P_{I}$

$$
\frac{z_{n-1}}{x_{n-1}+x_{n}}+\frac{z_{n}}{x_{n}+x_{n+1}}=-x_{n}^{2}+\gamma, \quad \gamma=(\lambda / 2)^{2}-2 \mu \eta
$$


with the initial conditions $x_{0}=\eta \beta_{0}+\lambda / 2, x_{1}=\eta \beta_{1}+\lambda / 2$.

Proof. Set $A(x)=a_{0}$, where $a_{0}$ is a non-zero constant. Eq. (31) yields (39), with $l_{n}(0)=$ $\eta \gamma_{n+1}+\mu$, thus, we have (44).

Let us now look at Eq. (17), $A=\left(x-\beta_{n}\right)\left(l_{n}-l_{n-1}\right)+\Theta_{n}-\gamma_{n} \frac{\Theta_{n-2}}{\gamma_{n-1}}$, evaluated at $x=\beta_{n}$,

$$
a_{0}=\Theta_{n}\left(\beta_{n}\right)-\gamma_{n} \frac{\Theta_{n-2}\left(\beta_{n}\right)}{\gamma_{n-1}} .
$$

Using the formulae in Lemma 3.2 we get, after simplifications,

$$
a_{0}=-\gamma_{n+1}\left(\eta\left(\beta_{n}+\beta_{n+1}\right)+\lambda\right)+\gamma_{n}\left(\eta\left(\beta_{n-1}+\beta_{n}\right)+\lambda\right), \quad n \geq 1,
$$

where $\eta=2 b_{3}+c_{2}, \lambda=2 b_{3} \beta_{0}+2 b_{2}+c_{1}$. By writing (48) as

$$
T_{n+1}=T_{n}-a_{0}, \quad T_{n}=\gamma_{n}\left(\eta\left(\beta_{n-1}+\beta_{n}\right)+\lambda\right),
$$

iteration yields $T_{n+1}=-n a_{0}+T_{1}$, thus we obtain

$$
\gamma_{n+1}\left(\eta\left(\beta_{n}+\beta_{n+1}\right)+\lambda\right)=-n a_{0}+\gamma_{1}\left(\eta\left(\beta_{0}+\beta_{1}\right)+\lambda\right),
$$

hence, we obtain (45).

Using the equivalent form of (45),

$$
\gamma_{n+1}=\frac{-n a_{0}+\gamma_{1}\left(\eta\left(\beta_{0}+\beta_{1}\right)+\lambda\right)}{\eta \beta_{n}+\eta \beta_{n+1}+\lambda}
$$

into (44), we obtain the second order difference equation

$$
\eta \frac{-(n-1) a_{0}+\gamma_{1}\left(\eta\left(\beta_{0}+\beta_{1}\right)+\lambda\right)}{\eta \beta_{n-1}+\eta \beta_{n}+\lambda}+\eta \frac{-n a_{0}+\gamma_{1}\left(\eta\left(\beta_{0}+\beta_{1}\right)+\lambda\right)}{\eta \beta_{n}+\eta \beta_{n+1}+\lambda}=-\eta \beta_{n}^{2}-\lambda \beta_{n}-2 \mu .
$$

By multiplying the above equation by $\eta$ we get

$$
\begin{aligned}
\eta^{2} \frac{-(n-1) a_{0}+\gamma_{1}\left(\eta\left(\beta_{0}+\beta_{1}\right)+\lambda\right)}{\eta \beta_{n-1}+\eta \beta_{n}+\lambda}+ & \eta^{2} \frac{-n a_{0}+\gamma_{1}\left(\eta\left(\beta_{0}+\beta_{1}\right)+\lambda\right)}{\eta \beta_{n}+\eta \beta_{n+1}+\lambda} \\
& =-\left(\eta^{2} \beta_{n}^{2}+\lambda \eta \beta_{n}+(\lambda / 2)^{2}\right)+(\lambda / 2)^{2}-2 \mu \eta
\end{aligned}
$$

Thus, by defining $x_{n}, z_{n}$ as in (46), the above equation reads as (47).

Remark 3.7. Moreover, using (44) with $n=0$ and (30), we get the following condition on $\beta_{0}$ :

$$
4 b_{3}^{2}+c_{2}\left(b_{1}+c_{2}+2 b_{2} \beta_{0}\right)+b_{3}\left(c_{2}\left(4+\beta_{0}^{2}\right)-2 c_{1} \beta_{0}-2 c_{0}\right)=0 .
$$

\subsection{Further results and generalizations}

Another way to prove Theorem 3.3 and to generalize to the case $A(x)=x-\alpha$, where $\alpha$ is an arbitrary parameter (recall that $\alpha=0$ in Theorem 3.3) is by symbolic computations, 
which can be performed in any computer algebra system, for instance, Mathematica ${ }^{1}$. Indeed, assuming the form

$$
x_{n}=\frac{k_{1}}{k_{2} \beta_{n}+k_{3}}, \quad y_{n}=k_{4} \gamma_{n}+k_{5}(n)
$$

and

$$
x_{n-1} x_{n}=\frac{y_{n}-k_{6}(n)}{y_{n}^{2}-\xi_{0}}, \quad y_{n}+y_{n+1}=\frac{1}{x_{n}}\left(k_{7}-\frac{1}{x_{n}}\right),
$$

where only $k_{5}$ and $k_{6}$ are the functions of $n$ and others are constants, and substituting into (13) and (15), we obtain the following theorem.

Theorem 3.8. Let $S$ be a Stieltjes function satisfying $A S^{\prime}=B S^{2}+C S+D$ with

$$
\begin{gathered}
A(x)=x-\alpha, B(x)=b_{3} x^{3}+b_{2} x^{2}+b_{1} x+b_{0}, \\
C(x)=c_{2} x^{2}+c_{1} x+c_{0}, D(x)=d_{1} x+d_{0},
\end{gathered}
$$

where $d_{1}$ and $d_{0}$ are given by (21) and (22), respectively. Let $\left\{P_{n}\right\}_{n \geq 0}$ be the SMOP associated with $S$, satisfying the recurrence relation (4),

$$
P_{n+1}(x)=\left(x-\beta_{n}\right) P_{n}(x)-\gamma_{n} P_{n-1}(x), \quad n=0,1,2, \ldots
$$

The corresponding equations (13) and (15) are solved by

$$
\begin{gathered}
x_{n}=\frac{k_{1}\left(2 b_{3}+c_{2}\right)}{k_{2}\left(2 b_{2}+2 \alpha b_{3}+c_{1}+\alpha c_{2}+2 b_{3} \beta_{0}\right)+\beta_{n}\left(2 b_{3}+c_{2}\right)}, \\
y_{n}=\frac{k_{2}^{2}\left(2 n+2 b_{1}+c_{0}+2 b_{2} \beta_{0}+2 b_{3}\left(\beta_{0}^{2}+\gamma_{1}\right)+2\left(2 b_{3}+c_{2}\right) \gamma_{n}+g_{1}\right)}{2\left(2 b_{3}+c_{2}\right) k_{1}^{2}},
\end{gathered}
$$

satisfying

$$
x_{n-1} x_{n}=\frac{2\left(k_{2}^{2}\left(2 n+2 b_{1}+c_{0}+2 b_{3} \beta_{0}+2 b_{3}\left(\beta_{0}^{2}+\gamma_{1}\right)+g_{2}\right)-2\left(2 b_{3}+c_{2}\right) k_{1}^{2} y_{n}\right)}{\left(2 b_{3}+c_{2}\right) k_{1}^{2}\left(c_{0}^{2}+4 b_{0}\left(b_{2}+c_{1}+\left(2 b_{3}+c_{2}\right) \beta_{0}\right)-4 y_{n}^{2}\right)}
$$

and

$$
y_{n}+y_{n+1}=\frac{k_{2} x_{n}\left(2 b_{2}+c_{1}+2 b_{3} \beta_{0}+2 \alpha\left(2 b_{3}+c_{2}\right)\right)-\left(2 b_{3}+c_{2}\right) k_{1}}{\left(2 b_{3}+c_{2}\right) k_{1} x_{n}^{2}} .
$$

Here, $\left.g_{1}=\alpha^{2}\left(2 b_{3}+c_{2}\right)+\alpha\left(2 b_{2}+c_{1}+2 b_{3} \beta_{0}\right)\right), g_{2}=\alpha^{2}\left(2 b_{3}+c_{2}\right)+\alpha\left(2 b_{2}+c_{1}+2 b_{3} \beta_{0}\right)$, and $k_{1}, k_{2}$ are constants. The initial conditions are given by

$$
x_{0}=\frac{\left(2 b_{3}+c_{2}\right) k_{1}}{k_{2}\left(2 b_{2}+c_{1}+4 b_{3} \beta_{0}+c_{2} \beta_{0}+\alpha\left(2 b_{3}+c_{2}\right)\right)}
$$

and $y_{0}$, which is obtained from the formulae above with $n=0$.

${ }^{1}$ www.wolfram.com 
Note that by taking $k_{2}=\eta k_{1} / \sqrt{-\eta}$, where $\eta=2 b_{3}+c_{2}$ and $\alpha=0$, we obtain case (a) in Theorem 3.3, and similarly for case (b).

Remark 3.9. The technique used in Theorems 3.3 and 3.8 can not be extended to the case $\operatorname{deg}(A) \geq 2$. Indeed, if $\operatorname{deg}(A) \geq 2$, then in the right-hand side of (39) there appear additional sequences $\eta_{n}$ and $\nu_{n}$ (cf. (29)), as the formulae for $\ell_{n, 0}$ involve the coefficients $a_{3}, a_{2}$ of $A$ (cf. (25)). Such a fact that does not allow a reduction to the system of types $(35),(37)$ or $(49)-(50)$.

\section{Examples}

There are several families of orthogonal polynomials related to the Stieltjes function satisfying $A S^{\prime}=B S^{2}+C S+D$ such that the previous Theorems apply. Some of them are as follows.

\subsection{Example 1.}

Let us start by considering the SMOP $\left\{P_{n}\right\}_{n \geq 0}$ with respect to a modified Laguerre weight

$$
w(x)=|x|^{\alpha} e^{\alpha_{2} x^{2}+\alpha_{1} x}, x \in \mathbb{R}
$$

where $\alpha>-1, \alpha_{2}<0$. The Stieltjes function of $w$, here denoted by $\tilde{S}$, satisfies

$$
\tilde{A} \tilde{S}^{\prime}=\tilde{C} S+\tilde{D}
$$

with

$$
\tilde{A}(x)=x, \tilde{C}(x)=2 \alpha_{2} x^{2}+\alpha_{1} x+\alpha, \tilde{D}(x)=-2 \alpha_{2} x-2 \alpha_{2} \beta_{0}-\alpha_{1} .
$$

Let us now consider the sequence of associated polynomials of the first kind $\left\{P_{n}^{(1)}\right\}_{n \geq 0}$, and let $S^{(1)}$ denote its Stieltjes function. In account of $(7), S^{(1)}$ is Laguerre-Hahn of class $s=1$, as it satisfies

$$
x\left(S^{(1)}\right)^{\prime}=B\left(S^{(1)}\right)^{2}+C S^{(1)}+D
$$

with

$$
B=\gamma_{1} \tilde{D}, C=-\tilde{C}-2\left(x-\beta_{0}\right) \tilde{D}, \quad D=\frac{1}{\gamma_{1}}\left(x+\left(x-\beta_{0}\right) \tilde{C}+\left(x-\beta_{0}\right)^{2} \tilde{D}\right)
$$

that is,

$$
\begin{aligned}
& B(x)=b_{1} x+b_{0}, \quad b_{1}=-2 \gamma_{1} \alpha_{2}, b_{0}=-\gamma_{1}\left(2 \alpha_{2} \beta_{0}+\alpha_{1}\right) \\
& C(x)=c_{2} x^{2}+c_{1} x+c_{0}, \quad c_{2}=2 \alpha_{2}, c_{1}=\alpha_{1}, c_{0}=-\alpha-2 \alpha_{1} \beta_{0}-4 \alpha_{2} \beta_{0}^{2}, \\
& D(x)=d_{1} x+d_{0}, \quad d_{1}=\frac{1}{\gamma_{1}}\left(1+\alpha+\alpha_{1} \beta_{0}+2 \alpha_{2} \beta_{0}^{2}\right), d_{0}=-\frac{\beta_{0}}{\gamma_{1}}\left(\alpha+\alpha_{1} \beta_{0}+2 \alpha_{2} \beta_{0}^{2}\right) .
\end{aligned}
$$


The sequences

$$
x_{n}=\frac{\sqrt{-2 \alpha_{2}}}{2 \alpha_{2} \beta_{n}+c_{1}}, \quad y_{n}=-\left(2 \alpha_{2} \gamma_{n}+n+\mu\right), \quad \mu=-2 \alpha_{2} \gamma_{1}-\alpha / 2-\alpha_{1} \beta_{0}-2 \alpha_{2} \beta_{0}^{2}
$$

satisfy $(36)$,

$$
x_{n-1} x_{n}=\frac{y_{n}+n+\mu}{y_{n}^{2}-\xi_{0}}, \quad y_{n}+y_{n+1}=\frac{1}{x_{n}}\left(\frac{\alpha_{1}}{\sqrt{-2 \alpha_{2}}}-\frac{1}{x_{n}}\right)
$$

with $\xi_{0}=\frac{1}{4} C^{2}(0)-B(0) D(0)$.

Remark 4.1. Orthogonal polynomials with respect to the semi-classical weight (53) with $\alpha_{2}=-1$ and $\alpha_{1}=t$, being $t$ some time parameter, were studied in [31], showing that the sequences

$$
x_{n}=\frac{\sqrt{2}}{-2 \beta_{n}+t}, \quad y_{n}=2 \gamma_{n}-n-\alpha / 2
$$

(cf. (35)) satisfy

$$
x_{n-1} x_{n}=\frac{y_{n}+n+\alpha / 2}{y_{n}^{2}-\frac{1}{4} \alpha^{2}}, \quad y_{n}+y_{n+1}=\frac{1}{x_{n}}\left(\frac{t}{\sqrt{2}}-\frac{1}{x_{n}}\right)
$$

(cf. (36)). Also, in [22], it is shown that the coefficients in the three term recurrence relation, as functions of the parameter $t$, can be expressed in terms of Wronskians of parabolic cylinder functions that arise in the description of special function solutions of the fourth Painlevé equation.

\subsection{Example 2.}

Let us start by considering the SMOP $\left\{P_{n}\right\}_{n \geq 0}$ with respect to a modified Laguerre weight (see [32])

$$
w(x)=(x-\alpha)^{\nu} e^{b x^{2}+a x},
$$

where $b<0, a<0$. As $w$ satisfies the Pearson equation $(x-\alpha) w^{\prime}=\left(2 b x^{2}+(a-2 \alpha b) x+\right.$ $\nu-\alpha a) w$, the Stieltjes function of $w$, here denoted by $\tilde{S}$, satisfies

$$
\tilde{A} \tilde{S}^{\prime}=\tilde{C} S+\tilde{D}
$$

with

$$
\tilde{A}(x)=x-\alpha, \tilde{C}(x)=2 b x^{2}+(a-2 \alpha b) x+\nu-\alpha a, \tilde{D}(x)=-2 b x-2 b \beta_{0}-a+2 \alpha b
$$

The sequences

$$
x_{n}=\frac{2 k_{1} b}{a+2 b \beta_{n}}, \quad y_{n}=\frac{2 n+\nu+4 b \gamma_{n}}{4 b k_{1}^{2}}
$$


satisfy (51) and (52) with $k_{2}=1$,

$$
x_{n-1} x_{n}=\frac{2 n+\nu-4 b k_{1}^{2} y_{n}}{b k_{1}^{2}\left((\nu-\alpha a)^{2}-4 y_{n}^{2}\right)}, \quad y_{n}+y_{n+1}=\frac{a+2 \alpha b x_{n}-2 b k_{1}}{2 b k_{1} x_{n}^{2}} .
$$

Let us now consider the sequence of associated polynomials of the first kind $\left\{P_{n}^{(1)}\right\}_{n \geq 0}$, and let $S^{(1)}$ denote its Stieltjes function. In account of $(7), S^{(1)}$ is Laguerre-Hahn of class $s=1$, as it satisfies

$$
(x-\alpha)\left(S^{(1)}\right)^{\prime}=B\left(S^{(1)}\right)^{2}+C S^{(1)}+D
$$

with

$$
B=\gamma_{1} \tilde{D}, C=-\tilde{C}-2\left(x-\beta_{0}\right) \tilde{D}, D=\frac{1}{\gamma_{1}}\left(x-\alpha+\left(x-\beta_{0}\right) \tilde{C}+\left(x-\beta_{0}\right)^{2} \tilde{D}\right),
$$

that is,

$$
\begin{aligned}
& B(x)=b_{1} x+b_{0}, b_{1}=-2 b \gamma_{1}, b_{0}=\gamma_{1}\left(2 \alpha b-2 \beta_{0} b-a\right), \\
& C(x)=c_{2} x^{2}+c_{1} x+c_{0}, c_{2}=2 b, c_{1}=a-2 \alpha b, c_{0}=-\nu+\alpha a+2 \beta_{0}\left(2 \alpha b-2 \beta_{0} b-a\right), \\
& D(x)=d_{1} x+d_{0}, d_{1}=\frac{1}{\gamma_{1}}\left(1+\nu-\alpha a-\beta_{0}\left(-a+2 \alpha b-2 \beta_{0} b\right),\right. \\
& d_{0}=\frac{1}{\gamma_{1}}\left(-\alpha-\beta_{0}(\nu-\alpha a)+\beta_{0}^{2}\left(-2 b \beta_{0}-a+2 \alpha b\right) .\right.
\end{aligned}
$$

The sequences

$$
x_{n}=\frac{2 b}{a+2 b \beta_{n}}, \quad y_{n}=\frac{2 n-\nu-4 \gamma_{1} b+2\left(\alpha-\beta_{0}\right)\left(a+2 b \beta_{0}\right)+4 b \gamma_{n}}{4 b}
$$

satisfy (51) and (52) with $k_{1}=k_{2}=1$,

$$
\begin{gathered}
x_{n-1} x_{n}=\frac{4 b y_{n}+4 \gamma_{1} b-2 n+\nu-2\left(\alpha-\beta_{0}\right)\left(a+2 b \beta_{0}\right)}{b\left(\nu+2 \beta_{0}\left(a+2 b \beta_{0}\right)-\alpha\left(a+4 b \beta_{0}\right)\right)^{2}-4 \gamma_{1}\left(a+2 b\left(\beta_{0}-\alpha\right)\right)^{2}-4 y_{n}^{2}}, \\
y_{n}+y_{n+1}=\frac{(a+2 \alpha b) x_{n}-2 b}{2 b x_{n}^{2}} .
\end{gathered}
$$

Remark 4.2. Note that in [32] only a third order nonlinear difference equation for the semi-classical Laguerre polynomials related to (56) is derived, and hence the results of this subsection are, to the authors' best knowledge, new in the literature.

\subsection{Remarks}

Let us remark that when (53) reduces to $w(x)=x^{\alpha} e^{-x}, \quad \alpha>2, \quad x>0$, an integral representation for the linear functional corresponding to $S^{(1)}$ is known. In such a case, $\left\{P_{n}^{(1)}\right\}_{n \geq 0}$ is orthogonal with respect to the linear functional defined by [33]

$$
\langle u, p(x)\rangle=\int_{0}^{\infty} \frac{p(x) x^{\alpha} e^{-x} d x}{\left|\psi\left(\alpha, 1-\alpha, x e^{-\pi i}\right)\right|^{2}}, \quad \alpha>2,
$$


where

$$
\begin{gathered}
\psi(a, b, x)=1+\sum_{n=1}^{+\infty} \frac{(a)_{n}}{(b)_{n}} x^{n}, \\
(a)_{n}=a(a+1) \cdots(a+n-1), \quad n \geq 1, \quad(a)_{0}=1 .
\end{gathered}
$$

In general, the integral representation of the Laguerre-Hahn linear functionals is an open problem. Another open problem (see [16]) is to determine all the Stieltjes functions, say $S^{1}$, satisfying a Riccati equation such as (55), which are obtained by a rational spectral transformation of $S$ satisfying (54), that is, $S^{1}=\frac{a S+b}{c S+d}$, where $a, b, c, d$ are polynomials.

\section{Acknowledgements}

The manuscript was substantially improved thanks to the suggestions and comments of an anonymous referee. The authors are greatly indebt to her $/$ him.

The work of M.N. Rebocho was partially supported by the Centre for Mathematics of the University of Coimbra UID/MAT/00324/2013, funded by the Portuguese Government through FCT/MEC and co-funded by the European Regional Development Fund through the Partnership Agreement PT2020.

The partial support of G. Filipuk by the Alexander von Humboldt Foundation is acknowledged.

The authors thank KU Eichstaett-Ingolstadt for hospitality.

\section{References}

[1] J. Dini, Sur les formes linéaires et les polynômes orthogonaux de Laguerre-Hahn [doctoral dissertation]. Paris (France): University Pierre et Marie Curie; 1988.

[2] Marcellán F, Prianes E. Orthogonal polynomials and Stieltjes functions: the Laguerre-Hahn case. Rend. Mat. Appl. 1996;16:117-141.

[3] Maroni P. Une théorie algébrique des polynômes orthogonaux. Application aux polynômes orthogonaux semi-classiques. In: Brezinski C, Gori L, Ronveaux A, editors. Orthogonal polynomials and their applications. Baltzer, Basel: IMACS Ann. Comput. Appl. Math. 9; 1991. p. $95-130$.

[4] Ismail MEH, Letessier J, Valent G. Linear birth and death models and associated Laguerre polynomials. J. Approx. Theory. 1988;56:337-348.

[5] Marcellán F, Ronveaux A. Co-recursive orthogonal polynomials and fourth order differential equation. J. Comput. Appl. Math. 1989;25:105-109.

[6] Ronveaux A. Fourth-order differential equations for the numerator polynomials. J. Phys. A.: Math. Gen. 1988;21:749-753.

[7] Magnus AP. Riccati acceleration of the Jacobi continued fractions and Laguerre-Hahn polynomials. In: Werner H, Bunger HT, editors. Padé Approximation and its Applications. Lect. Notes in Math. 1071, Berlin: Springer Verlag; 1984. p. 213-230.

[8] Magnus AP. Painlevé-type differential equations for the recurrence coefficients of semiclassical orthogonal polynomials. J. Comput. Appl. Math. 1995;57:215-237.

[9] Van Assche W. Discrete Painlevé equations for recurrence coefficients of orthogonal polynomials. In: Elaydi S, Cushing J, Lasser R, Ruffing A, Papageorgiou V, Van Assche W, editors. Difference equations, special functions and orthogonal polynomials. Hackensack, NJ: World Scientific; 2007. p. 687-725. 
[10] Alaya J. Quelques resultats nouveaux dans la théorie des polynômes de Laguerre-Hahn [doctoral dissertation]. Tunis (Tunisia): University of Tunis II; 1996.

[11] Bouakkaz H, Maroni P. Description des polynômes orthogonaux de Laguerre-Hahn de classe zéro. In: Brezinski C, Gori L, Ronveaux A, editors. Orthogonal polynomials and their applications. Baltzer, Basel: IMACS Ann. Comput. Appl. Math. 9; 1991. p. 189-194.

[12] Dehesa JS, Marcellán F, Ronveaux A. On orthogonal polynomials with perturbed recurrence relations. J. Comput. Appl. Math. 1990;30:203-212.

[13] Ronveaux A, Van Assche W. Upward extension of the Jacobi matrix for orthogonal polynomials. J. Approx. Theory. 1996;86:335-357.

[14] Branquinho A, Paiva A, Rebocho MN. Sylvester equations for Laguerre-Hahn orthogonal polynomials on the real line. Appl. Math. Comput. 2013;219:9118-9131.

[15] Dueñas H, Marcellán F, Prianes E. Perturbations of Laguerre-Hahn functional: modification by the derivative of a Dirac delta. Integral Transforms Spec. Funct. 2009;20:59-77.

[16] Zhedanov A. Rational spectral transformations and orthogonal polynomials. J. Comput. Appl. Math. 1997;85:67-86.

[17] Belmehdi S. On semi-classical linear functionals of class $s=1$. Classification and integral representations. Indag. Math. 1992;3:253-275.

[18] Belmehdi S, Ronveaux A. Laguerre-Freud's equations for the recurrence coefficients of semiclassical orthogonal polynomials. J. Approx. Theory. 1994;76:351-368.

[19] Dominici D, Marcellán F. Discrete semiclassical orthogonal polynomials of class one. Pacific J. Math. 2014;268:389-411.

[20] Foupouagnigni M, Hounkonnou MN, Ronveaux A. Laguerre-Freud equations for the recurrence coeffcients of $D_{w}$ semi-classical orthogonal polynomials of class one. J. Comput. Appl. Math. 1998;99:143-154.

[21] Magnus AP. On Freud's equations for exponential weights. J. Approx. Theory. 1986;46:6599.

[22] Clarkson PA, Jordaan K. The relationship between semiclassical Laguerre polynomials and the fourth Painlevé equation. Constr. Approx. 2014;39:223-254.

[23] Filipuk G, Van Assche W, Zhang L. The recurrence coefficients of semi-classical Laguerre polynomials and the fourth Painlevé equation. J. Phys. A: Math. Theor. 2002;45:205201 (13 pp).

[24] Alaya J, Maroni P. Symmetric Laguerre-Hahn forms of class $s=1$. Integral Transforms Spec. Funct. 1996;4:301-320.

[25] Szegö G. Orthogonal polynomials. 4th ed. Providence Rhode Island: Amer Math Soc Colloq Publ.; 1975.

[26] Chihara TS. An Introduction to Orthogonal Polynomials. Gordon and Breach, New York; 1978.

[27] Marcellán F, Rocha IA. On semiclassical linear functionals: integral representations. J. Comput. Appl. Math. 1995;57:239-249.

[28] Marcellán F, Rocha IA, Complex path integral representation for semiclassical linear functionals. J. Approx. Theory. 1998;94:107-127.

[29] Chen Y, Ismail MEH. Ladder operators and differential equations for orthogonal polynomials. J. Phys. A: Math. Gen. 1997;30:7817-7829.

[30] Maroni P. An introduction to second degree forms. Adv. Comput. Math. 1995;3:59-88.

[31] Boelen L, Van Assche W. Discrete Painlevé equations for recurrence coefficients of semiclassical Laguerre polynomials. Proc. Amer. Math. Soc. 2010;138:1317-1331.

[32] Spicer PE, Nijhoff FW. Semi-classical Laguerre polynomials and a third-order discrete integrable equation. J. Phys. A: Math. Theor. 2009;42:454019 (9pp).

[33] Askey R, Wimp J. Associated Laguerre and Hermite polynomials. Proc. Roy. Soc. Edin. 1984;96:15-37. 